\title{
Influência da adiposidade nas respostas fisiológicas e afetivas na caminhada de ritmo autosselecionado
}

\author{
Influence of adiposity on physiological and affective responses \\ in walking at a self-selected pace
}

\author{
Kleverton Krinski \\ Hassan Mohamed Elsangedy \\ Cosme Franklin Buzzachera \\ Heriberto Colombo \\ Bruno Vinicius Santos \\ Wagner de Campos \\ Sérgio Luiz Carlos dos Santos \\ Sergio Gregório DaSilva
}

1 Universidade Federal do Paraná. Departamento de Educação Física. Curitiba, PR. Brasil

Recebido em 20/09/08 Revisado em 02/03/09 Aprovado em 21/04/09
Resumo - O objetivo desse estudo foi verificar a influência da adiposidade corporal sobre as respostas fisiológicas e afetivas durante caminhada em ritmo autosselecionado. Foram investigadas 45 mulheres adultas, sedentárias, que foram divididas de acordo com o seu percentual de gordura corporal em baixo tercil (BT, n=15), médio tercil (MT, n=15) e alto tercil (AT, n=15). Todos os participantes foram submetidos a duas sessões experimentais, as quais foram compostas de (I) uma pré-avaliação inicial, avaliação antropométrica e teste incremental máximo em esteira, e (II) um teste de 20-minutos de caminhada em ritmo autosselecionado. As respostas fisiológicas $\left(\dot{V} \mathrm{O}_{2}\right.$ e FC) foram mensuradas, continuamente, durante toda a realização do teste de 20-minutos de caminhada em ritmo autosselecionado, enquanto a resposta afetiva foi determinada somente a cada intervalo de 5 minutos do teste. Para a análise estatística, empregaram-se análises de variância de um fator (adiposidade corporal), adotando $\mathrm{P}<0,05$. Nenhuma diferença significativa foi verificada, na velocidade de caminhada autosselecionada, entre os grupos experimentais $\left(1,63,1,60\right.$, e 1,47 m.seg ${ }^{-1}$ para BT, MT e AT, respectivamente), enquanto o $\% \dot{V} \mathrm{O}_{2 \max }$ foi significativamente maior em AT $(61,8 \%)$, comparado à MT $(55,8 \%)$ e BT $(52,8 \%)(\mathrm{P}<0,05)$. Por outro lado, a resposta afetiva $(2,36,1,80$, e 1,85 para BT, MT, e AT, respectivamente) foi similar entre os grupos experimentais. Com base nos resultados expostos acima, sugere-se que a adiposidade corporal pode influenciar as respostas fisiológicas, porém não a resposta afetiva, durante caminhada em ritmo autosselecionado.

Palavras-chave: Caminhada; Adiposidade; Afeto.

Abstract - The aim of this study was to investigate the influence of adiposity on physiological and affective responses during walking at a self-selected pace. Forty-five sedentary women were divided into three groups according to their percentage of body fat (low tertile, LT, $n=15$; medium tertile, MT, $n=15$; and high tertile, HT, n=15). Each participant completed a maximal exercise test and a 20-min bout of treadmill walking at a self-selected pace. The preferred walking speed

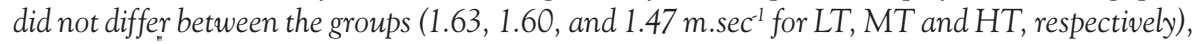
whereas $\% \dot{V} \mathrm{O}_{2 \max }$ was higher in HT $(61.8 \%)$ compared to MT $(55.8 \%)$ and LT $(52.8 \%)(P<.05)$. Nevertheless, affective $(2.36,1.80$, and 1.85 for $L T, M T$ and HT, respectively) response were similar between the groups. These findings suggest that adiposity did influence physiological, but not affective responses, during treadmill walking at a self-selected pace in sedentary women.

Key words: Walking; Adiposity; Affect. 


\section{INTRODUÇÃO}

A prescrição de atividades de intensidade vigorosas tem sido apontada como um potencial fator contribuinte para a falta de aderência em programas de exercício físico ${ }^{1-3}$. Dados oriundos de estudos epidemiológicos e de intervenção demonstraram que indivíduos previamente sedentários tendem a apresentar uma maior probabilidade de aderir a prescrições de atividades de baixa-moderada intensidade ${ }^{4}$, comparadas a atividades de maior intensidade ${ }^{5}$. Embora esses estudos tenham reforçado a idéia de que a intensidade do esforço influencia a aderência a programas de exercício físico, quaisquer informações adicionais a respeito dos mediadores dessa relação não foram providas.

Recentemente, o grau de prazer individual durante realização de exercício físico tem sido considerado um provável mediador da relação entre intensidade de esforço e aderência ${ }^{3,5-7}$. Desse modo, isso poderia, logicamente, indicar que as atividades capazes de proporcionar um prazer individual apresentam uma maior probabilidade de sucesso relativo à aderência, em comparação àquelas atividades incapazes de proporcionar esse prazer individual ${ }^{3}$.

O uso da autosseleção da intensidade de exercício físico é considerado um meio adequado para produzir efeitos positivos sobre as respostas afetivas em indivíduos previamente sedentários ${ }^{3,4,8}$. Evidências de prévios estudos têm sugerido que as respostas fisiológicas ao exercício físico em um ritmo preferido podem ser influenciados por alguns fatores, dentre eles o nível de adiposidade corporal ${ }^{9,10}$. Basicamente, esses estudos revelam que os sujeitos com maiores níveis de adiposidade tendem a autosselecionar uma velocidade de caminhada mais lenta, demonstrando uma similar ou mesmo maior resposta fisiológica a sujeitos com menores níveis de adiposidade, em razão de uma acentuada diminuição da capacidade funcional motora a qual sujeitos obesos são expostos, em virtude da redução da aptidão de desempenho aeróbio e anaeróbio muscular ${ }^{11}$.

Desse modo, uma vez que indivíduos com uma maior adiposidade corporal são mais predispostos a exibir uma baixa tolerância a elevadas intensidades de exercício físico ${ }^{9}$, essa similar ou maior resposta fisiológica à atividade em ritmo autosselecionado poderia ter um impacto negativo sobre a sua aderência.

Dessa maneira, torna-se lógico especular que o nível de adiposidade individual pode influenciar as respostas afetivas, também, durante exercício físico em ritmo autosselecionado. Entretanto, nenhum estudo foi conduzido para examinar os efeitos da adiposidade corporal sobre as respostas fisiológicas e afetivas durante exercício físico em um ritmo preferido. Neste sentido, o presente estudo teve por objetivo verificar a influência da adiposidade corporal sobre as respostas fisiológicas e afetivas, durante caminhada em ritmo autosselecionado, por mulheres adultas sedentárias.

\section{PROCEDIMENTOS METODOLÓGICOS}

\section{Sujeitos}

Participaram do presente estudo, 45 mulheres adultas, entre 20 e 45 anos de idade. $O$ recrutamento inicial das possíveis participantes foi realizado através de anúncios impressos, fixados em murais nos campus da Universidade Federal do Paraná (UFPR). Foram estabelecidos os seguintes critérios de inclusão: (a) condição de previamente sedentária, indicado por uma participação inferior a 30 minutos de atividade física moderada em três ou mais dias da semana ${ }^{12}$ (b) totalidade das respostas negativas ao Physical Activity Readiness Questionnaire (PARQ); (c) nenhum histórico de distúrbios cardiovasculares, respiratórios, musculoesqueléticos e metabólicos; e (d) nenhum histórico de tabagismo. O presente estudo foi aprovado pelo Comitê de Ética em Pesquisa da UFPR ( $n^{\circ}$ 477.127.07.11).

No intuito de melhor investigar a influência da adiposidade corporal sobre as respostas fisiológicas e afetivas, durante caminhada em ritmo autosselecionado, as participantes foram categorizadas como: baixo (BT $\leq 26,34 \%, n=15)$, médio (MT, $>26,34$ $\mathrm{e} \leq 30,94 \%, n=15$ ) e alto tercil (AT, $>30,94 \%, n$ $=15$ ), de acordo com o seu percentual de gordura corporal (\% gordura corporal).

\section{Avaliação Antropométrica}

A avaliação antropométrica foi realizada por um único pesquisador, previamente treinado. As variáveis, massa corporal (MC, em kg.; balança marca Toledo ${ }^{\circledR}$, modelo 2096, São Paulo, Brasil) e estatura (EST, em cm; estadiômetro marca Sanny ${ }^{\circledR}$, modelo Standard, São Bernardo do Campo, Brasil) foram obtidas de acordo com os procedimentos descritos por Lohman et al. ${ }^{13}$. O Índice de Massa Corporal (IMC, em $\mathrm{kg} \cdot \mathrm{m}^{-2}$ ) foi calculado como a massa corporal dividida pela estatura ao quadrado. A densidade corporal foi estimada pelo método de espessura de dobras cutâneas, de acordo com a equação proposta por Durnin e Womersley ${ }^{14}$. Posteriormente, o \% gordura corporal foi obtido mediante a equação de $\operatorname{Siri}^{15}$. 


\section{Sessão de Familiarização}

A sessão de familiarização consistiu de uma caminhada inicial em esteira (modelo XFit7, Reebok Fitness $^{\mathrm{TM}}$, Londres, Reino Unido) sem inclinação, por 5 minutos, no ritmo selecionado pelo sujeito. Após 10 minutos de repouso em posição sentada, esse procedimento de caminhada era então repetido. Todos os participantes foram instruídos a autosselecionar um ritmo, definido como uma velocidade que o sujeito considerou confortável para, no tempo estipulado, realizar a atividade ${ }^{10}$. Durante a sessão de familiarização e a sessão experimental, o marcador de velocidade da esteira foi, inicialmente, ajustado em 1,11 m.seg ${ }^{-1}\left(4,0 \mathrm{~km} \cdot \mathrm{h}^{-1}\right)$, sendo este visor ocultado para os sujeitos e somente visível para o avaliador ${ }^{10}$.

\section{Teste Incremental Máximo}

O teste incremental máximo foi realizado de acordo com o protocolo de Lind et $\mathrm{al}^{4}$. Inicialmente, os sujeitos executaram um aquecimento padrão (caminhada em esteira por $5 \mathrm{~min}$, em uma velocidade de $\left.1,11 \mathrm{~m} \cdot \mathrm{seg}^{-1}\right)$. Após $1 \mathrm{~min}$ de repouso, em posição sentada, o teste foi iniciado em uma velocidade de $1,11 \mathrm{~m} \cdot \mathrm{seg}^{-1}\left(4.0 \mathrm{~km} \cdot \mathrm{h}^{-1}\right)$ e sem inclinação, por $2 \mathrm{~min}$. Subsequentemente, a velocidade foi aumentada por $0,18 \mathrm{~m} \cdot \mathrm{seg}^{-1}\left(0.64 \mathrm{~km} \cdot \mathrm{h}^{-1}\right)$ sem inclinação, a cada 2 min, até exaustão volitiva ${ }^{4}$. $\mathrm{O}$ critério para atingir o $\dot{V} \mathrm{O}_{2 \max }$ deveria atender um dos seguintes critérios: (a) um platô no $\dot{V} \mathrm{O}_{2}$ (variações de $<150 \mathrm{~mL} \cdot \mathrm{min}^{-1}$ nas últimas três médias consecutivas de 20-seg); (b) uma RTR $\geq 1,10$; e (c) uma FC max $_{\text {dentro de } 10}$ bp. min $^{-1}$ da FC máxima predita pela idade. $O$ limiar ventilatório (LV) foi determinado pelo método de equivalente ventilatório, considerada a intensidade no qual o primeiro aumento súbito no equivalente ventilatório de oxigênio $\left(\dot{V} \mathrm{E} / \mathrm{O}_{2}\right)$ sem alterações no equivalente ventilatório de dióxido de carbono $(\dot{V}$ $\left.\mathrm{E} / \mathrm{CO}_{2}\right)^{16}$. Uma avaliação a posteriori para determinar o LV foi conduzida por dois experientes avaliadores, sendo determinado como LV o primeiro ponto de quebra no qual houve concordância nas identificações, não havendo concordância, um terceiro pesquisador foi consultado.

A FC (bp.min $\left.{ }^{-1}\right)$ foi mensurada, a cada 5 seg, durante todo o teste, usando um sistema de monitoramento Polar (Polar Electro ${ }^{\mathrm{TM}}$ F-5, Oy, Finlândia). Um sistema metabólico-respiratório de

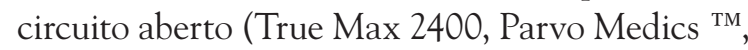
Salt Lake City, EUA) foi usado para mensurar $\dot{V} \mathrm{O}_{2}$, produção de dióxido de carbono $\left(\dot{V} \mathrm{CO}_{2}\right)$ e ventilação pulmonar ( $\dot{V}$ E, STPD), a cada 20 seg, durante todo o teste. Anteriormente a cada determinação do $\dot{V} \mathrm{O}_{2 \max }$ o analisador de gases foi calibrado com concentrações conhecidas de gases.

\section{Teste de 20 minutos de Caminhada em Ritmo Autosselecionado}

A sessão experimental foi composta de um teste de caminhada contínua, em esteira, sem inclinação, por 20 minutos. Inicialmente, os sujeitos realizaram um aquecimento padrão (caminhada em esteira, por $5 \mathrm{~min}$, em uma velocidade de $1,1 \mathrm{~m} \cdot \mathrm{seg}^{-1}$ ). Após 1 minuto de repouso, em posição sentada, o teste iniciou com velocidade de $1,11 \mathrm{~m} . \mathrm{seg}^{-1}$ (4,0 $\mathrm{km} \cdot \mathrm{h}^{-1}$ ), sem inclinação, por 2 minutos. Durante os próximos 3 min do teste de caminhada de 20 min, modificações na velocidade da esteira foram permitidas ad libitum. Subsequentemente, foram permitidas modificações na velocidade da esteira somente a cada 5 min do teste de 20-min de caminhada (min 5:00, 10:00 and 15:00).

As respostas metabólicas respiratórias e FC foram mensuradas, a cada $1 \mathrm{~min}$, durante o teste, usando os mesmos instrumentos e procedimentos do teste incremental máximo. Entretanto, somente os valores médios do último minuto de cada estágio foram considerados para análise dos dados fisiológicos (i.e., $\dot{V} \mathrm{O} 2$ and FC): (a) $\min 5$, (b) $\min 10$, (c) $\min 15$, and (d) $\min 20^{4}$.

\section{Mensurações da resposta afetiva.}

A escala de afeto de Hardy e Rejeski ${ }^{17}$ foi empregada para mensurar a resposta afetiva de prazer/desprazer. Essa escala apresenta uma medida bipolar de 11 pontos, variando de -5 à +5 , com descritores verbais entre "muito ruim" a "muito bom", com um ponto zero "neutro". A escala de afeto foi administrada durante os últimos $15 \mathrm{seg}$, de cada estágio de $2 \mathrm{~min}$, do teste incremental máximo e a cada $5 \mathrm{~min}$, do teste de 20 min de caminhada em ritmo autosselecionado. Os participantes foram questionados para indicar a sua sensação de prazer e desprazer nesses momentos. Definições padronizadas de afeto e uma série de informações foram apresentadas antes do teste incremental máximo e 20 min de caminhada em ritmo autosselecionado.

\section{Análise estatística}

Dados descritivos foram apresentados como média (M) \pm desvio-padrão (DP). Uma ANOVA de um fator (adiposidade) foi usada para examinar diferenças entre os grupos, nas respostas fisiológica e afetivas, durante teste de 20 min de caminhada em ritmo autosselecionado. Além disso, uma ANOVA $3 \times 4$ (adiposidade $\times$ tempo) de medidas repetidas 
foi usada para determinar as mudanças através do tempo e entre grupos, na respostas fisiológicas e afetivas, durante o teste de 20 min de caminhada em ritmo autosselecionado. Efeitos principais e de interação foram melhor analisados através de uma ANOVA de um fator com correções de Bonferroni $(P<0,05)$. Foi realizado a priori um cálculo de tamanho amostral e poder estatístico, adotando um alfa de 0,05; poder de 0,95 e tamanho de efeito 0,25 o qual demonstrou que seriam necessários 15 sujeitos para cada grupo. Todos os dados foram analisados usando SPSS 13.0 for Windows (SPSS, Inc., Chicago, USA).

\section{RESULTADOS}

As características demográficas, antropométricas, fisiológicas e afetivas dos sujeitos dos três grupos experimentais são listadas na Tabela 1. A ANOVA de um fator indicou efeitos significantes da adiposi- dade para a massa corporal $\left(F_{2,42}=2,468, P=0,001\right)$, $\operatorname{IMC}\left(F_{2,42}=20,567, P=0,001\right)$ e \% gordura corporal $\left(F_{2,42}=78,977, P=0,001\right)$. Contudo, nenhum efeito da adiposidade foi verificado para idade e estatura $(P>0,05)$, e outra variável $(P>0,05)$, exceto $\dot{V} \mathrm{O}_{2 \text { max }}$ $\left(F_{2,42}=11,804, P=0,001\right)$. Além disso, o $\dot{V} \mathrm{O}_{2 \max }(\mathrm{mL}$. $\left.\mathrm{kg}^{-1} \cdot \mathrm{min}^{-1}\right)$ foi maior para o grupo BT, comparado aos grupos experimentais MT e AT e o consumo de oxigênio no limiar ventilatório foi maior para o grupo BT, em comparação ao grupo AT $\left(F_{2,42}=\right.$ $5,014, P=0,01$ ).

As respostas fisiológicas e afetivas durante o teste de 20-minutos de caminhada em esteira, em ritmo autosselecionado são listadas na Tabela 2. A ANOVA one-way não indicou efeito significante da adiposidade em todas as variáveis analisadas $(P>0,05)$, com exceção do $\% \dot{V} \mathrm{O}_{2 \max }\left(F_{2,42}=3,978\right.$, $P=0,05)$. De um modo similar, nenhum efeito significante da adiposidade foi verificado na resposta afetiva $\left(F_{2,41}=0,537, P=0,589\right)$.

Tabela 1. Características demográficas, antropométricas, fisiológicas e afetivas dos participantes do estudo.

\begin{tabular}{|c|c|c|c|c|c|c|c|c|c|}
\hline & \multicolumn{3}{|c|}{$\mathrm{BT}(n=15)$} & \multicolumn{3}{|c|}{$\mathrm{MT}(n=15)$} & \multicolumn{3}{|c|}{ AT $(n=15)$} \\
\hline & $M$ & \pm & DP & $M$ & \pm & DP & $M$ & \pm & DP \\
\hline Idade (anos) & 28,1 & \pm & 5,7 & 28,8 & \pm & 6,4 & 32,4 & \pm & 4,8 \\
\hline EST $(\mathrm{cm})$ & 161,2 & \pm & 4,5 & 162,8 & \pm & 5,7 & 160,0 & \pm & 6,5 \\
\hline IMC $\left(\mathrm{kg} \cdot \mathrm{m}^{-2}\right)$ & 21,5 & \pm & 2,1 & 24,3 & \pm & $2,5^{*}$ & 28,7 & \pm & $4,2^{\#+}$ \\
\hline$\dot{V} \mathrm{O}_{2 \mathrm{LV}}$ & 27,3 & \pm & 6,2 & 24,3 & \pm & 4,7 & 21,1 & \pm & 4,9 \# \\
\hline $\mathrm{FC}_{\max }\left(\mathrm{b} \cdot \mathrm{min}^{-1}\right)$ & 190 & \pm & 14 & 184 & \pm & 10 & 181 & \pm & 8 \\
\hline $\mathrm{FC}_{\mathrm{LV}}$ & 154 & \pm & 14 & 151 & \pm & 13 & 148 & \pm & 14 \\
\hline$\dot{V} \mathrm{E}\left(\mathrm{L} \cdot \mathrm{min}^{-1}\right)$ & 61,9 & \pm & 9,9 & 61,7 & \pm & 10,7 & 60,1 & \pm & 10,1 \\
\hline
\end{tabular}

MC: Massa corporal; EST: Estatura; IMC: Índice de Massa Corporal: $\operatorname{VO}_{2 \max }$ : consumo máximo de oxigênio; $\mathrm{FC}_{\max }$ : frequência cardíaca máxima; $V$ E: ventilação pulmonar; RTR: razão de troca respiratória; LV: limiar ventilatório.* BT significativamente diferente de MT $(\mathrm{P}<0,05)$. " BT significativamente diferente de AT $(\mathrm{P}<0,05) .{ }^{+} \mathrm{MT}$ significativamente diferente de $\mathrm{AT}(\mathrm{P}<0,05)$.

Tabela 2. Respostas fisiológicas e afetivas durante teste de 20-minutos de caminhada em esteira, em ritmo autosselecionado.

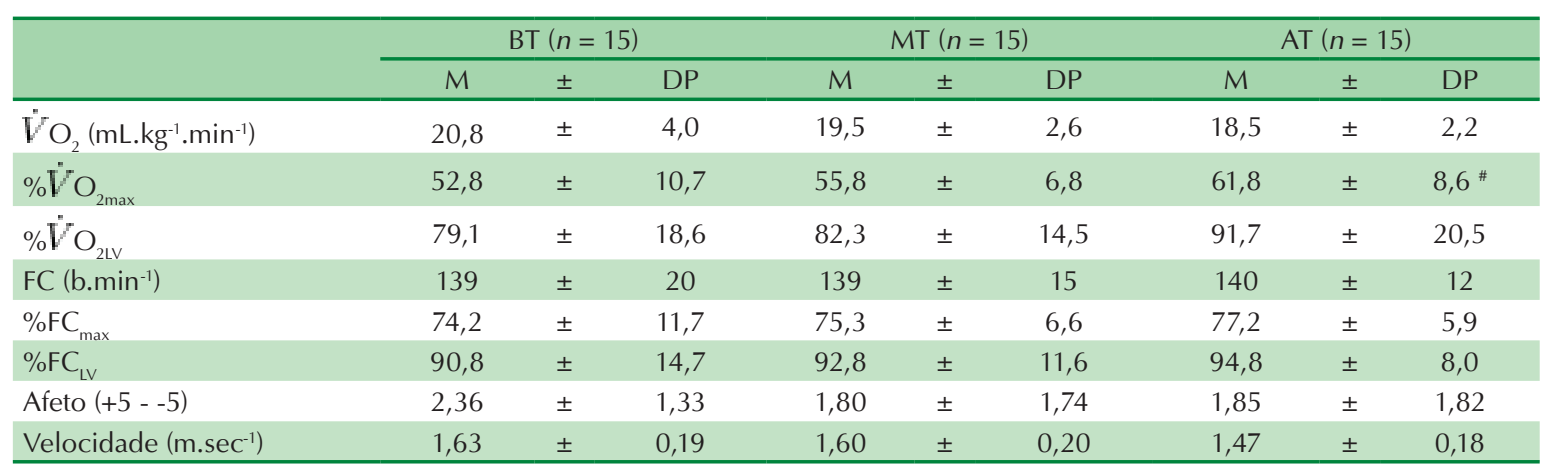

$\dot{V} \mathrm{O}_{2 \mathrm{lv}}$ : consumo de oxigênio; $\dot{V} \mathrm{O}_{2 \mathrm{max}}$ : consumo máximo de oxigênio; $\mathrm{FC}$ : frequência cardíaca; $\mathrm{FC}_{\max }$ : frequência cardíaca máxima; $\mathrm{LV}$ : limiar ventilatório. ${ }^{*} \mathrm{BT}$ significativamente diferente de MT $(\mathrm{P}<0,05)$. ${ }^{*} \mathrm{BT}$ significativamente diferente de AT $(\mathrm{P}<0,05) .{ }^{+} \mathrm{MT}$ significativamente diferente de AT $(\mathrm{P}<0,05)$ 
As respostas fisiológicas ( $\mathrm{VO}_{2}$ e FC) durante todos os 20-minutos do teste de caminhada em esteira, em ritmo autosselecionado são apresentadas nas Figuras 1 e 2. A ANOVA de medidas repetidas revelou efeitos principais significantes do tempo para $\mathrm{o} \% \dot{V} \mathrm{O}_{2 \max }\left(F_{1.88,26.38}=17,011, P=0,001 ; \eta_{\mathrm{p}}^{2}=0,549\right)$ e $\% \mathrm{FC}_{\text {max }}\left(F_{1.89,24.67}=32,911, P=0,001 ; \eta_{\mathrm{p}}^{2}=0,717\right)$. Por outro lado, nenhum efeito significante da adiposidade $\left(F_{2,28}=2,372, P=0,112 ; \eta_{p}^{2}=0,145\right.$ e $F_{2,26}=$ $0,861, P=0,435 ; \eta_{\mathrm{p}}^{2}=0,062$ para $\% V \mathrm{O}_{2 \max } \mathrm{e} \% \mathrm{FC}_{\text {max }}$, respectivamente) e interação adiposidade $\times$ tempo $\left(F_{3.42,47.93}=2,028, P=0,115 ; \eta_{p}^{2}=0,127\right.$ e $F_{4.03,52.50}=$ $2,346, P=0,066 ; \eta_{\mathrm{p}}^{2}=0,153$ para $\% \dot{V} \mathrm{O}_{2 \max }$ e $\% \mathrm{FC}_{\max }$ respectivamente) foi verificado. Esses dados revelam que o $\% \dot{V} \mathrm{O}_{2 \max }$ e $\% \mathrm{FC}_{\max }$ aumentam significativamente através do tempo com relação ao minuto 5 , independentemente da adiposidade corporal.

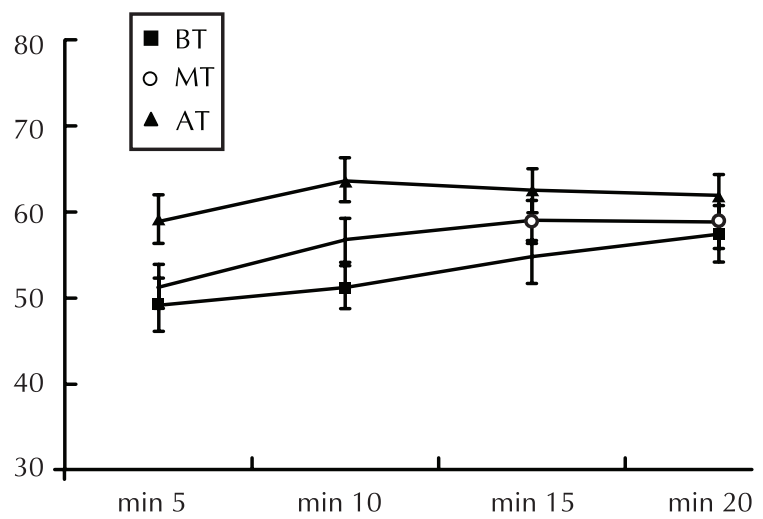

Figura 1. Resposta do $\% \dot{V} \mathrm{O}_{2 \max }$ durante o teste de 20 minutos de caminhada em esteira em ritmo autosselecionado. ${ }^{*} \mathrm{p}<$ 0,05 segundo ANOVA $3 \times 4$

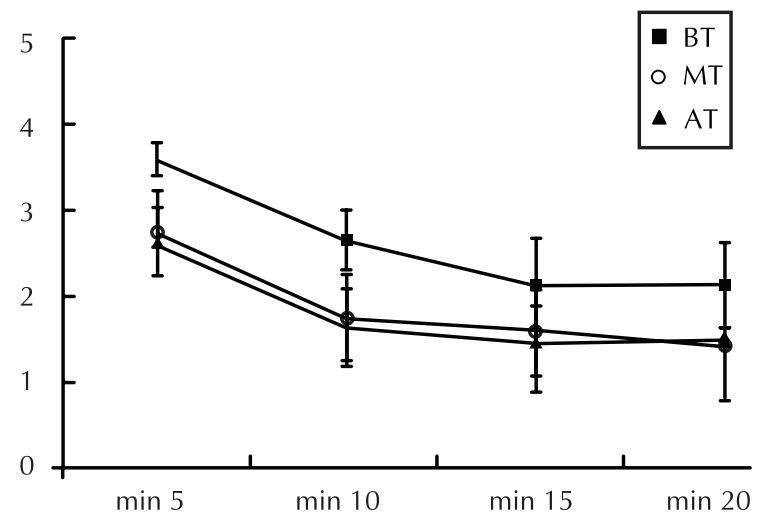

Figura 2. Resposta do \% $\mathrm{FC}_{\text {máx }}$ durante o teste de 20 minutos de caminhada em esteira em ritmo autosselecionado. ${ }^{*} \mathrm{p}<0,05$ segundo ANOVA $3 \times 4$

A resposta afetiva durante todos os 20 minutos do teste de caminhada em ritmo autosselecionado é apresentada na Figura 3. A ANOVA de medidas repetidas revelou efeitos significantes do tempo para o afeto $\left(F_{1.42,18.50}=11,174, P=0,001 ; \eta_{p}^{2}=0,462\right)$. Por outro lado, nenhum efeito significante da adiposida- de $\left(F_{2,26}=0,394, P=0,678 ; \eta^{2}=0,029\right)$ para o afeto, $\mathrm{e}$ da interação adiposidade $\times$ tempo $\left(F_{3,42,44.49}=0,564\right.$, $P=0,664 ; \eta_{\mathrm{p}}^{2}=0,042$ ), para o afeto, foi verificado. De um modo similar às respostas fisiológicas, esses dados indicam que o grau de satisfação durante o exercício físico realizado diminuiu através do tempo, independente da adiposidade corporal, entretanto, permaneceu positivo.

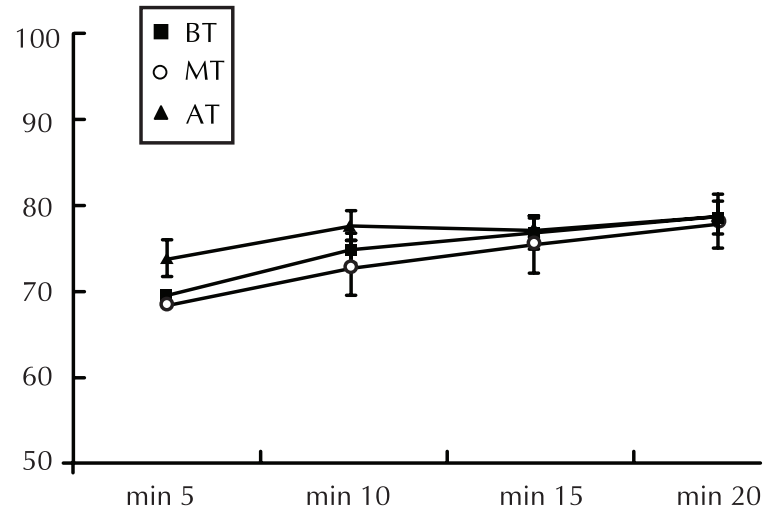

Figura 3. Respostas afetivas durante o teste de 20 minutos de caminhada, em esteira, em ritmo autosselecionado. ${ }^{*} \mathrm{p}<0,05$ segundo ANOVA $3 \times 4$

\section{DISCUSSÃO}

Os achados do presente estudo demonstraram que a velocidade autosselecionada de caminhada não diferiu entre os grupos experimentais $(P=0,082)$, porém as respostas fisiológicas $\left(\% \dot{V} \mathrm{O}_{2 \max }\right)$ foram, significativamente, maiores no grupo AT, comparadas àquelas dos grupos MT e BT $(P<0,05)$ (Tabela 2). Esses resultados corroboram estudos prévios $^{8,9}$. No estudo realizado por Ekkekakis e Lind $^{8}$, após avaliarem 9 mulheres não-obesas (21,6 $\pm 2,7 \%$ gordura corporal e IMC $\left.<25 \mathrm{~kg} \cdot \mathrm{m}^{-2}\right)$ e 16 obesas $(30,6 \pm 4,1 \%$ gordura corporal e IMC $>25$ $\mathrm{kg} \cdot \mathrm{m}^{-2}$ ) verificaram que aquelas com uma maior adiposidade corporal autosselecionaram uma velocidade de caminhada similar $\left(1,53 \pm 0,31 \mathrm{~m} \cdot \mathrm{seg}^{-1}\right)$ às mulheres com menor adiposidade corporal $(1,81$ $\left.\pm 0,55 \mathrm{~m} \cdot \mathrm{seg}^{-1}\right)$, mas elicitaram uma significante maior resposta fisiológica $(61-70 \%$ vs $48-65 \% \dot{V}$ $\mathrm{O}_{2 \max }$, respectivamente).

No presente estudo, torna-se relevante citar que as respostas fisiológicas $\left(\% \dot{V} \mathrm{O}_{2 \max }\right.$ e $\left.\% \mathrm{FC}_{\max }\right)$ na caminhada em esteira, em ritmo autosselecionado, gradualmente, aumentaram em relação aos 5 minutos iniciais de exercício físico, sem quaisquer mudanças posteriores (Figura 1 e 2). Esses achados são consistentes com prévios estudos ${ }^{1,4,8}$ e demonstram que os sujeitos não simplesmente selecionam uma singular taxa de trabalho, mas contrariamente, 
usam um tipo de estratégia de "pacing" (ou seja, ritmo). Os participantes desse estudo foram hábeis para autosselecionar um ritmo de caminhada capaz de produzir uma intensidade de exercício físico adequada para produzir benefícios à saúde ${ }^{12}$, independentemente da adiposidade corporal (Tabela 2). Esses resultados são similares àqueles de prévios estudos $^{4,8}$, porém estão em contraste àqueles de Mattsson et $\mathrm{al}^{9}$, que demonstraram que somente sujeitos com uma maior adiposidade corporal autosselecionam um ritmo de caminhada que resulta em uma intensidade capaz de produzir benefícios à saúde $\left(56 \% \dot{V} \mathrm{O}_{2 \max }\right)$, em comparação a sujeitos com uma menor adiposidade corporal $\left(36 \% \dot{V} \mathrm{O}_{2 \max }\right)$. Uma possível explicação para a discrepância entre os achados do estudo de Mattsson et $\mathrm{al}^{9}$, e os do presente estudo poderia estar relacionada às diferenças metodológicas na determinação do ritmo de caminhada preferido. Na presente investigação, todos os sujeitos autosselecionaram um ritmo de caminhada, durante um estímulo de 20 minutos de exercício físico, em uma esteira motorizada. Por outro lado, no estudo de Mattsson et $\mathrm{al}^{9}$, a velocidade de caminhada preferida dos sujeitos com uma menor adiposidade corporal foi derivada de um estudo de referência, e os sujeitos com uma maior adiposidade corporal foram avaliados, durante um estímulo de 4 minutos de exercício físico, em um corredor de 70-metros de comprimento.

O presente estudo reportou maiores respostas relativas ao consumo máximo de oxigênio (\% $\dot{V} \mathrm{O}_{2 \text { Máx }}$ ) no exercício físico, em ritmo preferido para os sujeitos do grupo AT (Tabela 2), os quais poderiam ser os maiores beneficiados por uma manutenção e/ou redução da adiposidade corporal. Contudo, as respostas fisiológicas relativas ao limiar ventilatório foram similares entre os grupos, com valores próximos ao $\operatorname{LV}\left(84,3 \pm 17,8 \%\right.$ do $\dot{V} \mathrm{O}_{2 \mathrm{LV}}$; $\left.92,8 \pm 11,4 \% \mathrm{FC}_{\mathrm{LV}}\right)$. Isso poderia ser importante uma vez que as maiores taxas de oxidação de gordura são verificadas em intensidades relativas, próximas ao limiar ventilatório ${ }^{12}$. Outro aspecto importante, envolvendo o LV é sua relação com as respostas afetivas, de acordo com Ekkekakis et al. ${ }^{5}$, o exercício físico realizado em intensidades abaixo do LV levam a respostas afetivas positivas, corroborando os achados da presente investigação.

Entretanto, baseado em prévios estudos, parecia lógico especular que essas maiores respostas fisiológicas ao exercício físico em ritmo preferido, reportadas pelo grupo AT, poderiam, também, induzir um decréscimo na resposta afetiva ${ }^{8}$, o qual poderia, por sua vez, ter um impacto negativo sobre a aderência em programas de exercício físico? Interessantemente, foi verificado que as respostas afetivas não diferiram entre os grupos experimentais (Tabela 2). Dessa maneira, poderia ser plausível que a magnitude das diferenças nas respostas fisiológicas ao exercício físico em ritmo autosselecionado, entre os grupos, não foi significante o bastante para influenciar a resposta afetiva. Além disso, corroborando prévios estudos ${ }^{3,8}$, sujeitos autosselecionaram um ritmo de caminhada que foi capaz de produzir uma resposta afetiva positiva, durante todo o teste de 20 minutos de exercício físico, independente da adiposidade corporal (Figura 3). Até o momento, esse é o primeiro estudo a avaliar a influência da adiposidade corporal sobre a resposta afetiva ao exercício físico em ritmo autosselecionado. A avaliação da resposta afetiva ("como" a pessoa sente-se) pode permitir uma descrição mais completa e melhor avaliação da experiência subjetiva ao exercício físico ${ }^{17}$.

Os dados do presente estudo devem ser extrapolados com cautela para diferentes populações (homens, sujeitos com elevada aptidão cardiorrespiratória e idosos), visto que foram utilizadas apenas mulheres sedentárias na composição da amostra. Uma limitação deste estudo foi a estimativa do \%gordura que foi determinada pelo método de dobras cutâneas. Embora esse método tenha sido empregado por muitos anos, o mesmo pode não ser tão acurado quanto os métodos mais recentes (ex., absorptiometria de raio-X de dupla energia, diluição de isótopos, entre outros) para a mensuração da gordura corporal. Apesar disso, a precisão do método de dobras cutâneas para a estimativa do $\%$ gordura corporal é reportado ser aceitável (ou seja, $+/-3,5 \%$ gordura corporal $)^{13}$, porém poderia ter influenciado a distribuição dos sujeitos entre os grupos AT e MT.

\section{CONCLUSÃO}

Pode-se concluir que as respostas fisiológicas, porém não a afetiva, são influenciadas pela adiposidade corporal, durante caminhada em esteira, em ritmo autosselecionado. $O$ presente estudo apresenta implicações teóricas e práticas. De uma perspectiva teórica, essa investigação provê informações úteis sobre o entendimento de como a adiposidade corporal individual influencia a autosseleção de um ritmo de exercício físico em suas respostas fisiológicas e também afetivas. Por outro lado, de um ponto de vista prático, os resultados desse estudo reforçam a utilidade da autosseleção de um ritmo de exercício 
físico como um estímulo fisiológico adequado para produzir benefícios à saúde em mulheres sedentárias, independente do seu nível de adiposidade corporal, proporcionando respostas afetivas positivas que, por sua vez, poderiam levar a uma maior taxa de aderência em programas de exercício físico, contribuindo, dessa forma, para a diminuição dos índices de sedentarismo e obesidade.

\section{REFERÊNCIAS BIBLIOGRÁFICAS}

1. Dishman RK. Advances in exercise adherence. Champaign: Human Kinetics; 1994.

2. Dishman RK, Buckworth J. Increasing physical inactivity: a quantitative synthesis. Med Sci Sports Exerc 1996;28(6):706-719.

3. Parfitt G, Rose EA, Burgess WM.. The psychological and physiological responses of sedentary individuals to prescribed and preferred intensity exercise. Br J Health Psychol 2006;11(1):39-53.

4. Lind E, Joens-Matre RR, Ekkekakis P. What intensity of physical activity do previously sedentary middle-aged women select? Evidence of a coherent pattern from physiological, perceptual and affective markers. Prev Med 2005;40(4):407-419.

5. Ekkekakis P, Hall EE, Petruzzello SJ. Variation and homogeneity in affective responses to physical activity of varying intensities: an alternative perspective on dose-response based on evolutionary considerations. J Sports Sci 2005;23(5):477-500.

6. Ekkekakis P, Hall EE, Petruzzello SJ. The relationship between exercise intensity and affective responses demystified: To crack 40-year-old nut, replace the 40-yearold nutcracker. Ann Behav Med 2008;35(2):136-149.

7. Williams DM, Dunsiger S, Ciccolo JT, Lewis BA, Albrecht AE, Marcus BH. Acute affective response to a moderate-intensity exercise stimulus predicts physical activity participation 6 and 12 months later. Psychol Sport Exerc 2008;9(3):231-245.

8. Ekkekakis P, Lind EE. Exercise does not feel the same when you are overweight: the impact of self-selected and imposed intensity on affect and exertion. Int J Obes 2006;23(4):477-500.
9. Matsson E, Evers LU, Rossner S. Is walking for exercise too exhausting for obese women? Int J Obes 1997;21(5):380-386.

10. Pintar JA, Robertson RJ, Kriska AM, Nagle E, Goss FL. The influence of fitness and body weight on preferred exercise intensity. Med Sci Sports Exerc 2006;38(5):981-988.

11. LaFortuna CL, Agosti F, Galli R, Busti C, Lazzer S, SArtorio $\mathrm{A}$. The energetic and cardiovascular response to treadmill walking and cycle ergometer in obese women. Eur J Appl Physiol 2008;103(6):707-717.

12. American College of Sports Medicine. Guidelines for exercise testing and prescription. Baltimore: Williams \& Wilkins; 2000.

13. Lohman TG, Roche AF, Martorell R. Anthropometric standardization reference manual. Champaign: Human Kinetics; 1988.

14. Durnin JVG, Womersley J. Body fat assessed from total body density and its estimation from skinfold thickness: measurements on 481 men and women aged from 16 to 72 years. Br J Nutr 1974;32(1):77-97.

15. Siri WE. Body composition from fluid space and density. In: Brozek J, Hanschel A, editors. Techniques for measuring body composition. Washington, DC: National Academy of Science 1961. p. 223-244.

16. Caiozzo VJ, Davis JA, Ellis JF, Azus JL, Vandagriff R, Prietto CA, et al. A comparison of gas exchange indices used to detect the anaerobic threshold. J Appl Physiol 1982;53(5):1184-1189.

17. Hardy CJ, Rejeski WJ. Not what, but how one feels: the measurement of affect during exercise. J Sports Exerc Psychol 1989;11(2):304-317.
Endereço para correspondência
Sergio Gregorio da Silva
Rua Coração de Maria, 92 - JD Botânico
80.215-370 - Curitiba, PR. Brazil
E-mail: sergiogregorio@ufpr.br 